\title{
The TAIGA timing array HiSCORE - first results
}

M. Tluczykont ${ }^{6, a}$, N.Budnev ${ }^{2}$, I.Astapov $^{9}$, N.Barbashina $^{9}$, A.Bogdanov $^{9}$, V.Boreyko $^{10}$, M.Brückner ${ }^{8}$, A.Chiavassa ${ }^{4}$, O.Chvalaev², O.Gress², T.Gress², O.Grishin ${ }^{2}$, A.Dyachok ${ }^{2}$, S.Epimakhov ${ }^{6}$, O.Fedorov ${ }^{2}$, A.Gafarov ${ }^{2}$, N.Gorbunov ${ }^{10}$, V.Grebenyuk ${ }^{10}$, A.Grinuk ${ }^{10}$, D.Horns $^{6}$, A.Kalinin ${ }^{10}$, N.Karpov ${ }^{1}$, N.Kalmykov', Y.Kazarina ${ }^{2}$, S.Kiryuhin ${ }^{2}$, R.Kokoulin ${ }^{9}$, K.Kompaniets $^{9}$, A.Konstantinov ${ }^{1}$, E.Korosteleva ${ }^{1}$, V.Kozhin ${ }^{1}$, E.Kravchenko ${ }^{11}$, M.Kunnas ${ }^{6}$, L.Kuzmichev ${ }^{1,2}$, Yu.Lemeshev², B.Lubsandorzhiev ${ }^{3}$, N.Lubsandorzhiev1, R.Mirgazov², R.Mirzoyan ${ }^{5,2}$, R.Monkhoev², R.Nachtigall ${ }^{6}$, E.Osipova1, A.Pakhorukov², M.Panasyuk ${ }^{1}$, L.Pankov², A.Petrukhin ${ }^{2}$, V.Poleschuk ${ }^{2}$, E.Popova ${ }^{1}$, A.Porelli ${ }^{8}$, E.Postnikov ${ }^{1}$, V.Prosin ${ }^{1}$, V.Ptuskin ${ }^{7}$, G.Rubtsov ${ }^{3}$, A.Pushnin ${ }^{2}$, V.Samoliga ${ }^{2}$, P.Satunin ${ }^{7}$, Yu.Semeney ${ }^{2}$, A.Silaev ${ }^{1}$, A.Silaev (junior) $^{1}$, A.Skurikhin ${ }^{1}$, M.Slunecka ${ }^{10}$, A.Sokolov ${ }^{11}$, C.Spiering ${ }^{8}$, L.Sveshnikova ${ }^{1}$, V.Tabolenko ${ }^{2}$, B.Tarashansky ${ }^{2}$, A.Tkachenko ${ }^{10}$, L.Tkachev ${ }^{10}$, D.Voronin ${ }^{2}$, R.Wischnewski ${ }^{8}$, A.Zagorodnikov ${ }^{2}$, V.Zurbanov ${ }^{2}$, D.Zhurov², and I.Yashin ${ }^{9}$

${ }^{1}$ Skobeltsyn Institute of Nuclear Physics MSU, Moscow, Russia

${ }^{2}$ Institute of Applied Physics, ISU, Irkutsk, Russia

${ }^{3}$ Institute for Nuclear Research of RAN, Moscow, Russia

${ }^{4}$ Dipartimento di Fisica Generale Universiteta di Torino and INFN, Torino, Italy

${ }^{5}$ Max-Planck-Institute for Physics, Munich, Germany

${ }^{6}$ Institut für Experimentalphysik, Universität Hamburg, Germany

${ }^{7}$ IZMIRAN, Moscow Region, Russia

${ }^{8}$ DESY, Zeuthen, Germany

${ }^{9}$ MEPhl: National Research Nuclear University MEPhl (Moscow Eng. Phys. Institute), Moscow, Russia

${ }^{10}$ JINR, Dubna, Russia

${ }^{11}$ Budker Institute of Nuclear Physics SB RAS, Novosibirsk State University, Novosibirsk, Russia

\begin{abstract}
Observations of gamma rays up to several $100 \mathrm{TeV}$ are particularly important to spectrally resolve the cutoff regime of the long-sought Pevatrons, the cosmic-ray PeV accelerators. One component of the TAIGA hybrid detector is the TAIGA-HiSCORE timing array, which currently consists of 28 wide angle $(0.6 \mathrm{sr})$ air Cherenkov timing stations distributed on an area of $0.25 \mathrm{~km}^{2}$. The HiSCORE concept is based on (nonimaging) air shower front sampling with Cherenkov light. First results are presented.
\end{abstract}

\section{Introduction}

The current generation of Imaging Air Cherenkov Telescopses (IACTs, e.g. H.E.S.S., MAGIC, and VERITAS) allowed the detection of more than 150 sources of very high energy $(\mathrm{E}>100 \mathrm{GeV})$ gamma rays [1]. The IACT technique is also used for the planned Cherenkov Telescope Array CTA [2]. Given the quick drop in flux with increasing energy, very large instrumented areas are required to access the

\footnotetext{
ae-mail: martin.tluczykont@physik.uni-hamburg.de
} 
ultra high energy (UHE, E $>10 \mathrm{TeV}$ ) regime. A cost effective method for the instrumentation of large areas with a wide field of view is the (non-imaging) shower-front timing technique. The weak point a poor gamma-hadron separation towards low energies - can be compensated by a hybrid combination with a small number of small sized IACTs, such as currently implemented by the TAIGA (Tunka Advanced Instrument for Gamma ray and cosmic-ray Astrophysics) experiment [3, 4].

The main astrophysical motivation of TAIGA is the measurement of the cutoff regime of known Galactic sources. Some of these sources might be cosmic-ray Pevatrons, i.e. cosmic-ray accelerators reaching up to the knee energies (around $3 \mathrm{PeV}$ proton energy). For more details see [5, 6].

\section{First results from the TAIGA timing array HiSCORE}

The experiment: TAIGA currently consists of 28 timing stations distributed over an area of $0.25 \mathrm{~km}^{2}$ in the Tunka valley in Siberia (see Fig. 1). This array is an implementation of the HiSCORE wide-angle timing array concept [7]. Each of the 28 existing TAIGA-HiSCORE stations consists of four 8 inchphotomultiplier tubes (PMTs), equipped with light concentrators, resulting in $0.5 \mathrm{~m}^{2}$ light collection area per station, and a field of view of $0.6 \mathrm{sr}$. The DAQ is based on fast GHz signal sampling [8]. Two independent time-calibration methods were sucessfully employed, reaching the required sub-ns accuracy $[8,9]$. The primary event parameters are reconstructed using signal timing and the amplitude [9-11]. Additionally, a small IACT (4.75 $\mathrm{m}$ dish diameter) is currently in commissioning and will be operated using a new hybrid approach, combining imaging and timing (see e.g. [12] and [13, 14]). The sensitivity of the current timing array alone is shown as a solid line in Figure 2. For comparison,

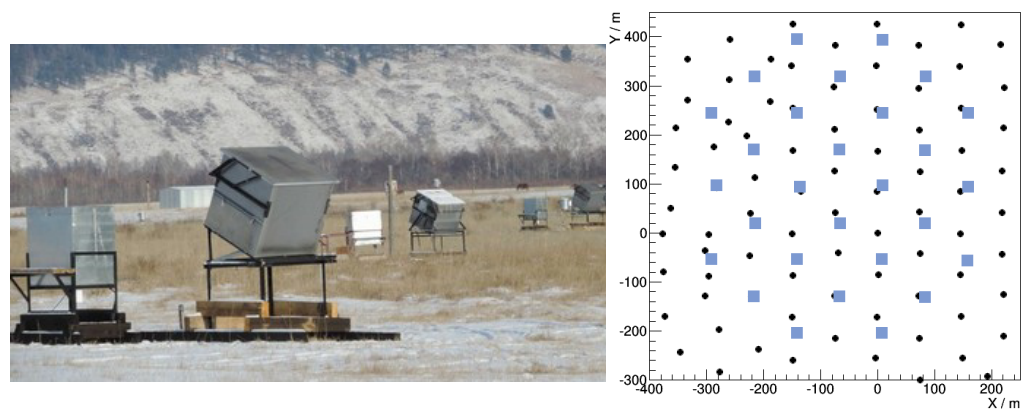

Figure 1. Left: photograph of 3 TAIGA timing array stations and their electronic boxes. Right: TAIGA timing array layout since 2014. The timing stations (previously HiSCORE) are shown in blue along with the Tunka-133 cosmic-ray array (black).

also the sensitivity of the combined TAIGA array is shown. More details on TAIGA and the new hybrid observation mode are given in [12].

Data-MC verification: from October 2015 to February 2016, $250 \mathrm{~h}$ of observation time were taken, containing $10^{7}$ air shower events. The individual station trigger rate ( 8 to $12 \mathrm{~Hz}$ ), as well as the 4-station-coincidence array rate $(10$ to $18 \mathrm{~Hz})$ could be verified using MC simulations, which reporduce the observed rates using a single station threshold of about 250 p.e. This translates to an energy threshold of $50 \mathrm{TeV}$. Further studies could verify the validity of the observed station multiplicity per event, as well as the angular resolution. The latter was studied using the chessboard method, dividing the array in subarrays, each providing a reconstructed event direction. The angular difference $\alpha$ between the reconstructed directions depends on the reconstruction quality and the number of stations used in each subarray. Applying this analysis to both, real data and (proton) MC simulations, the reconstruction quality of the current setup could be verified (see Fig. 3). The value of the angular resolution for gamma rays is $0.4^{\circ}$ at threshold and below $0.2^{\circ}$ above $100 \mathrm{TeV}$, as predicted in [11]. 


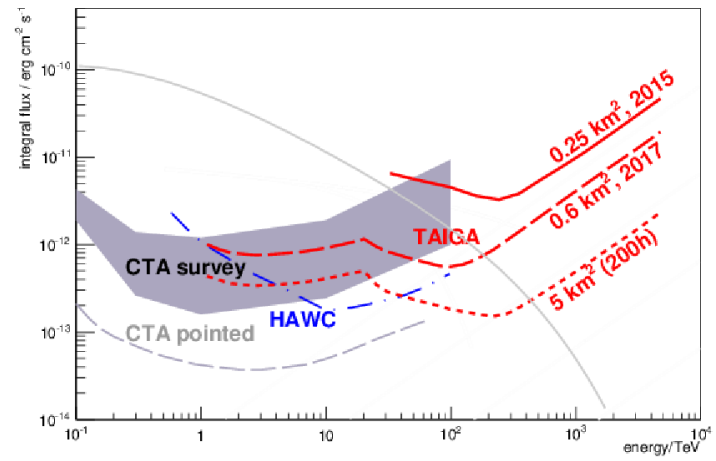

Figure 2. The sensitivity (after $200 \mathrm{~h}$ for the timing array and $50 \mathrm{~h}$ for the TAIGA IACTs) of the current $0.25 \mathrm{~km}^{2}$ TAIGA timing array (HiSCORE) and the planned hybrid stage, consisting of an extension of the timing array to $0.6 \mathrm{~km}^{2}$ in combination with two IACTs. In dotted red, the sensitivity is shown for $5 \mathrm{~km}^{2}$, including 10 IACTs. TAIGA curves are based on simulations and reconstruction methods presented in [7, 10, 11, 13]. A parametrization of the Crab Nebula flux by [15] is shown as a solid grey line. Figure adapted from [6], see references therein.

Crab Nebula data: using a standard ring background estimation method on blinded real data, the field of view was found to be free of artifacts of positive or negative fluctuations in excess of what is statistically expected for the test region of $10^{\circ} \times 10^{\circ}$. Performing the same analysis on the position of the Crab Nebula, a weak excess was found. Up to now the analysis was done without cut optimizations nor gamma-hadron separation. The observations are compatible with reasonable Crab Nebula flux extrapolations to higher energies and an instrumented area of $0.25 \mathrm{~km}^{2}$. We expect to reach better sensitivity optimizing the analysis, increasing the area as planned, and combining the timing array with IACTs.

A first source, the ISS: a recent serendipitous discovery, opens up opportunities for calibration and possibly different physics. Three intervals of $\sim 1 \mathrm{~s}$ duration with extremely high array trigger rate (few $\mathrm{kHz}$ as compared to the usual $15 \mathrm{~Hz}$ array rate) were found from November to February. These data are compatible with a $4 \mathrm{kHz}$ periodic light source, and are best described by a plane-wave time front shape, strikingly different from air shower events with a curved shower front. The reconstructed event directions are coincident with the trajectory of the International Space Station (ISS), passing almost vertically over the TAIGA observation site (see Fig. 3). As the strong light source, we identified the CATS-LIDAR [16] onboard the ISS, emitting $\sim 1 \mathrm{~mJ}$ at a wavelength of $532 \mathrm{~nm}$ at $4 \mathrm{kHz}$, and pointing almost vertically downwards at a few-degree inclination. This ISS light source is an interesting object for TAIGA calibration and atmospheric light scattering studies. Also other air Cherenkov installations like IACTs could benefit from this light source. A detailed analysis is underway.

\section{Summary}

As a part of the hybrid TAIGA array, the TAIGA timing array, based on the HiSCORE concept, is currently being operated with a total instrumented area of $0.25 \mathrm{~km}^{2}$. First studies of real data and comparisons to MC simulations show that the detector is reasonably well understood. While observations are compatible with expectations, in the current stage, no significant excess can be expected from the Crab Nebula. An unexpected result was achieved very recently, with the detection of a LIDAR onboard the ISS, which can be used for calibration measurements or other studies in future.

\section{Acknowledgements}

We acknowledge the support of the Russian Federation Ministry of Education and Science ( agreements N 14.B25.31.0010, zadanie No 3.889.2014/K ), the Russian Science Foundation (grant 15-12- 

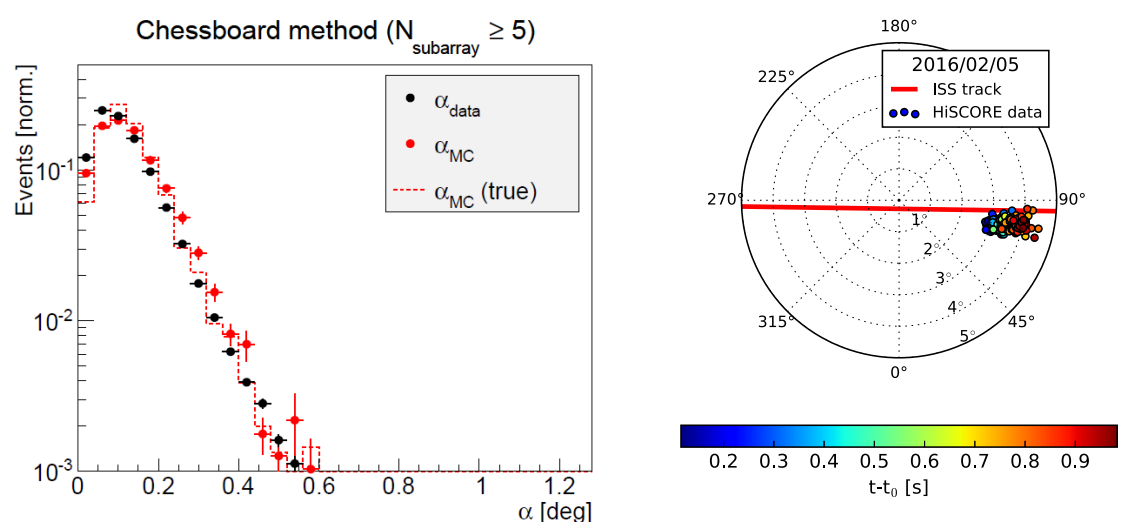

Figure 3. Left: angular resolution study with the chessboard method (see text), applied to data (black) and MC (red). Right: HiSCORE events (circles) reconstructed for the ISS passage on 5.2.2016 (color coded for registration time). The ISS track is calculated from NORAD ISS-TLE.

20022) the Russian Foundation for Basic research (15-02-10005, 15-02-05769, 16-02-00738, 16-2913035), the Helmholtz association (grant HRJRG-303), and the DFG (grant TL 51-3).

\section{References}

[1] S.P. Wakely, D. Horan, International Cosmic Ray Conference 3, 1341 (2008)

[2] B.S. Acharya, M. Actis, T. Aghajani, G. Agnetta, J. Aguilar et al., Astr.Part.Phys. 43, 3 (2013)

[3] R. Mirzoyan, 40th COSPAR Scientific Assembly 40 (2014)

[4] N. Budnev et al., Journal of Physics: Conference Series 718, 052006 (2016)

[5] M. Tluczykont, D. Hampf, D. Horns et al., Advances in Space Research 48, 1935 (2011)

[6] M. Tluczykont, N. Budnev, I. Astapov et al., Verlag Deutsches Elektronen-Synchrotron, Connectige Neutrino Physics and Astronomy pp. 135-142 (2016)

[7] M. Tluczykont, D. Hampf, D. Horns, D. Spitschan, L. Kuzmichev, V. Prosin, C. Spiering, R. Wischnewski, Astroparticle Physics 56, 42 (2014)

[8] O. Gress, I. Astapov, N. Budnev et al., NIMA, dx.doi.org/10.1016/j.nima.2016.08.031 (2016)

[9] A. Porelli, D. Bogorotskii, M. Brückner et al., J. of Phys: Conf. Ser. 632, 012041 (2015)

[10] S.F. Berezhnev, D. Besson, N.M. Budnev et al., NIMA 692, 98 (2012)

[11] D. Hampf, M. Tluczykont, D. Horns, NIMA 712, 137 (2013)

[12] L. Kuzmichev et al., These proceedings (2017)

[13] M. Kunnas, I. Astapov, N. Barbashina et al., Journal of Physics: Conference Series 632, 012040 (2015)

[14] M. Tluczykont, I. Astapov, N. Barbashina et al., Journal of Physics: Conference Series 632, 012042 (2015)

[15] M. Meyer, D. Horns, H.S. Zechlin, Astronomy and Astrophysics 523, A2 (2010)

[16] http://cats.gsfc.nasa.gov/ 